\title{
Strengthening and Softening of Nanoceramics: a Brief Review
}

\author{
A.G. Sheinerman ${ }^{1,2,3}$ and M.Yu. Gutkin ${ }^{1,2,4}$ \\ ${ }^{1}$ Institute of Problems of Mechanical Engineering, Russian Academy of Sciences, St. Petersburg 199178, Russia \\ ${ }^{2}$ Peter the Great St. Petersburg Polytechnic University, St. Petersburg 195251, Russia \\ ${ }^{3}$ Saint Petersburg State University, 7/9 Universitetskaya nab., St. Petersburg 199034, Russia \\ ${ }^{4}$ ITMO University, St. Petersburg 197101, Russia
}

Received: November 10, 2019

\begin{abstract}
We briefly review the experimental data, the results of computer simulations and analytical models that describe the mechanisms of room-temperature plastic deformation of nanocrystalline ceramics (NCCs) and the effects of these mechanisms on hardness of NCCs. We demonstrate the importance of grain boundary (GB) mediated processes, such as GB sliding, grain rotation and GB amorphization in NCCs. We show that these processes can be responsible for contradictive data on hardness of nanocrystalline $\mathrm{MgAl}_{2} \mathrm{O}_{4}$ spinel with small grain sizes, which demonstrate either direct or inverse Hall-Petch dependence for hardness.
\end{abstract}

High strength and hardness of nanocrystalline ceramics (NCCs) make them good candidates for the use in various industries, including electronics, optics, and power engineering [1-6]. Commonly, the hardness of ceramics obeys the Hall-Petch law, which predicts the linear relation between the microhardness and the inverse square root of grain size [7-9]. At the same time, several research groups [10-16] demonstrated the breakdown of the Hall-Petch dependence in nanocrystalline $\mathrm{ZrN}$, TiN, $\mathrm{TiO}_{2}, \mathrm{MgO}$, and $\mathrm{MgAl}_{2} \mathrm{O}_{4}$ below some critical grain size. Like many nanocrystalline metals (see the pioneering work [17] and reviews in [18-31]), below this critical grain size, nanocrystalline ceramics demonstrated an inverse Hall-Petch dependence, with a reduction in hardness with a decrease in grain size. In contrast to this, Muche et al. [32] and Yang et al. [33] recently succeeded in the synthesis of superhard nanocrystalline $\mathrm{MgAl}_{2} \mathrm{O}_{4}$ and $\mathrm{ZnAl}_{2} \mathrm{O}_{4}$ samples which showed a direct Hall-Petch dependence down to the grain size of 7 and $10 \mathrm{~nm}$, respectively. The reason for these contradictory results is not clear yet, and its explanation requires the understanding of the plastic deformation processes in nanoceramics.
In several research works plastic deformation of nanoceramics has been studied using large-scale molecular dynamics (MD) simulations. For example, Szlufarska et al. [34] carried out computer simulations of plastic deformation of nanocrystalline silicon carbide with a grain size of $8 \mathrm{~nm}$ under indentation. The simulation demonstrates that plastic deformation starts in $\mathrm{SiC}$ at amorphous GB regions through GB sliding and grain rotation (Fig. 1), as well as, to a small extent, through dislocation glide across grains.

An important role of GBs in the deformation of NCCs has also been confirmed by Feng et al. [35], who performed MD simulations of plastic deformation of nanocrystalline tungsten carbide (WC) under a uniaxial compressive load. The simulations demonstrated the transition from the direct to the inverse Hall-Petch relation for the yield strength at a critical grain size of $24 \mathrm{~nm}$. At grain sizes below the critical one, Feng et al. [35] observed no intragrain dislocation activity, and plastic deformation was realized through GB sliding and grain rotation (Fig. 2a). At a critical grain size, small intragrain dislocation activity was also observed (Fig. 2b). At grain sizes exceeding the critical one, plastic deformation of

Corresponding author: A.G. Sheinerman, email: asheinerman@ gmail.com 

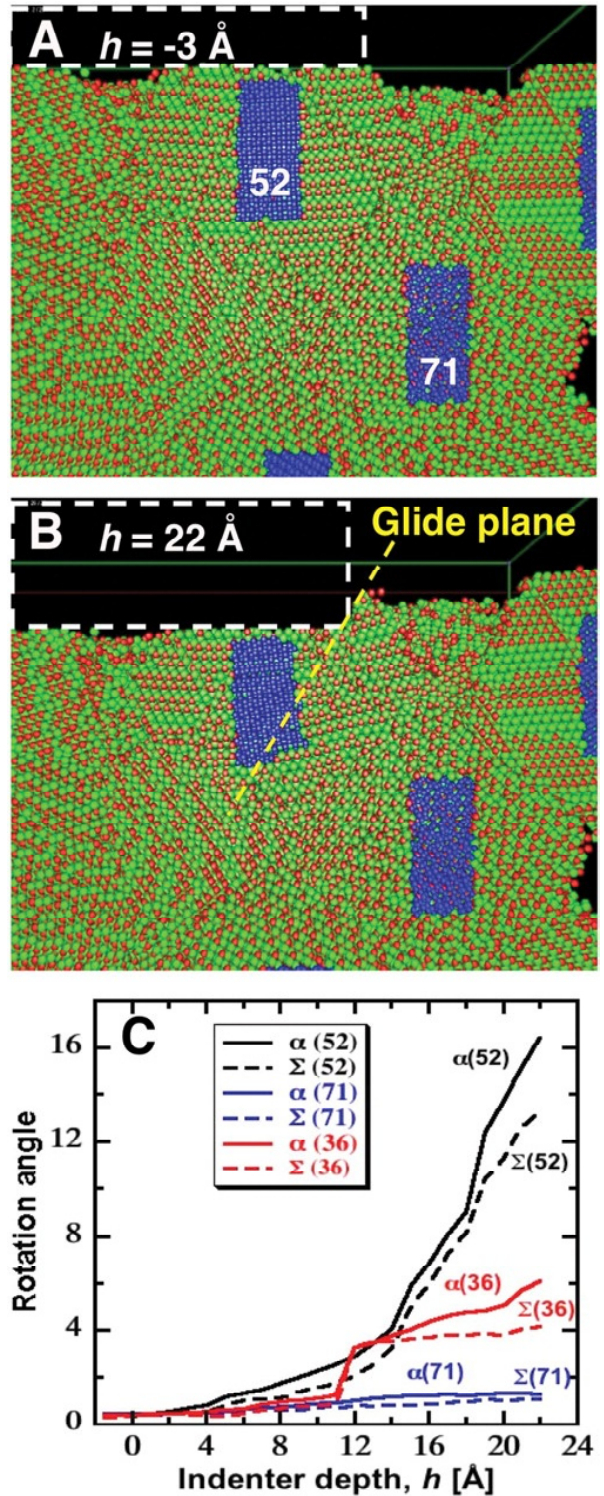

Fig. 1. Rotation of grains in nanocrystalline $\mathrm{SiC}$ under indentation revealed in MD simulations. (A and $\mathrm{B}$ ) $\mathrm{Si}$ (green) and $\mathrm{C}$ (red) atoms are shown for the cases of (A) purely elastic deformation and (B) just after the onset of yielding (white dashed lines depict the position of the indenter, $h$ is the indentation depth). Grain 52 experiences enormous shear and undergoes large rotation and deformation. (C) Rotation angle $\alpha(n)$ and its standard deviation $\Sigma(n)$ of selected grains with number $n=52,71$, and 36. Grain 52 undergoes large rotation and deformation (black curve). Localization of deformation is reflected in the lack of rotation of grain 71 (blue curve). Reproduced from Szlufarska et al. [34], with permission. Copyright (2005), American Association for the Advancement of Science.

tungsten carbide was realized via lattice dislocation motion across grains (Fig. 2c).

More recently, Guo et al. [16] carried out MD simulations and nanoindentation tests combined with
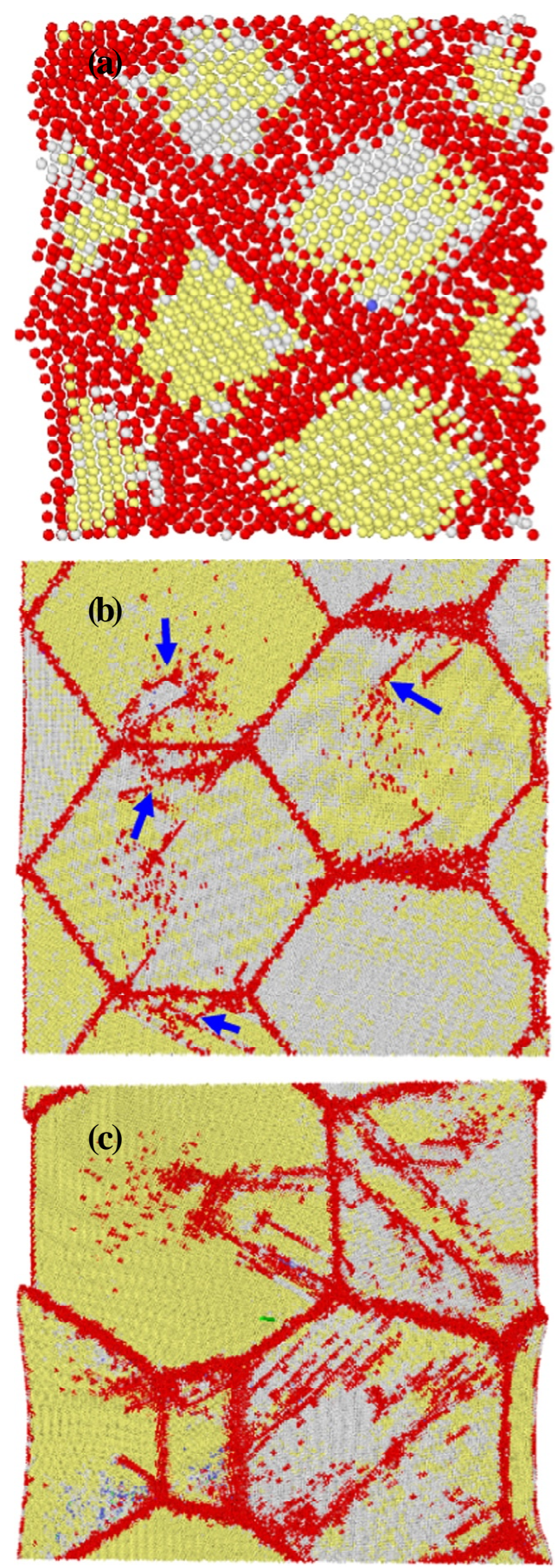

Fig. 2. The deformation microstructures at three representative grain sizes of polycrystalline WC: (a) $5.0 \mathrm{~nm}$, (b) $24.0 \mathrm{~nm}$ and (c) $31.4 \mathrm{~nm}$. The atoms (both at the grain boundaries and in the grain interiors) with higher potential are colored red, and the rest are in the HCP structure. The arrows in (b) show the sites of dislocation intersections. Reproduced from Feng et al. [35]. Copyright (2017), Institute of Physics Publishing.

transmission electron microscopy (TEM) of nanocrystalline boron carbide ceramics. In contrast to the simulations of nanocrystalline $\mathrm{SiC}$ [34], in the simulations [16] for $\mathrm{B}_{4} \mathrm{C}$ specimens, $\mathrm{GBs}$ of $\mathrm{B}_{4} \mathrm{C}$ initially 

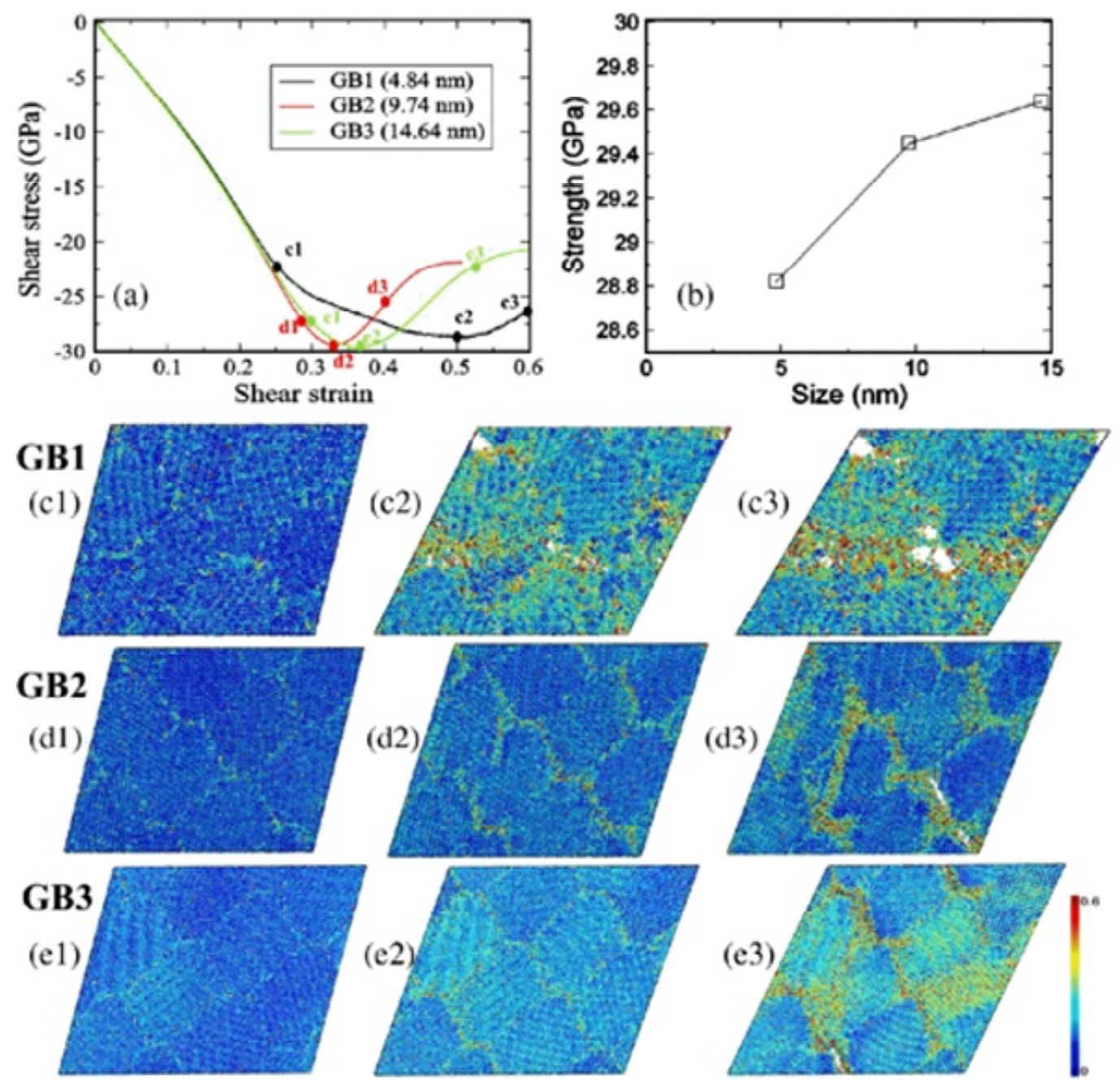

Fig. 3. (a) Stress-strain relationships of three GB models at finite shear deformation with shear rate of $0.1 \mathrm{ps}^{-1}$. (b) The shear strength of three GB models. (c1)-(e1) Snapshots of the beginning of plastic deformation for three GB models. (c2)-(e2) Snapshots of the end of plastic deformation, reaching the maximum shear strength. (c3)-(e3) Snapshots of the cavitation formation. Atoms are colored based on their atomic shear strain. The sizes of GB3 and GB2 are 3 and 2 times larger than the size of GB1. Reproduced from Guo et al. [16], with permission. Copyright (2018), American Physical Society, https://doi.org/10.1103/PhysRevLett.121.145504.

had crystalline structures. The simulations for the $\mathrm{B}_{4} \mathrm{C}$ specimens with the grain size of approximately 5,10 , and $15 \mathrm{~nm}$ demonstrated that plastic deformation in these specimens occurs via GB sliding, which starts at the values of the local shear strain of $0.25,0.275$, and 0.3 for the specimens with grain size of 5,10 and $15 \mathrm{~nm}$, respectively (Figs. 3a and 3c). This means that at a smaller grain size, plastic deformation starts at a smaller applied stress, implying an inverse Hall-Petch behavior. In the simulations, after some plastic deformation, the local shear stress in GBs reaches a maximum absolute value and then drops. The maximum in the shear stress - shear strain curve corresponds to the initiation of amorphization at GBs (Figs. 3a and 3c). The ultimate shear strength decreases with a decrease in the grain size (Fig. 3b). With further deformation, cavitation starts in the amorphous region (Fig. 3c), which eventually re- sults in the failure of the specimen. The formation of GB amorphous bands in nanocrystalline or ultrafine-grained $\mathrm{B}_{4} \mathrm{C}$ (with the grain size from 40 to $150 \mathrm{~nm}$ ) beneath the indenter surface has been directly observed using transmission electron microscopy (TEM) [16]. These observations also demonstrated the absence of any intragranular plastic deformation as well as the absence of cracks under the indenter. The authors of [16] suggested that the experimentally observed amorphous bands originated due to preceding GB sliding, in accord with the results of the atomistic simulations. At the same time, amorphization can also promote GB sliding, enhancing ductility of $\mathrm{B}_{4} \mathrm{C}$ [16]. The possible reason is that amorphization should lead to the delocalization of dislocations accumulated in triple junctions due to GB sliding, eliminating their back stresses and thereby facilitating plastic deformation in GBs. 
The plastic deformation processes in two $\mathrm{MgAl}_{2} \mathrm{O}_{4}$ specimens (with grain sizes of 22 and $43 \mathrm{~nm}$ ) under indentation have been experimentally studied in detail by Ratzker et al. [36]. They observed a high density of dislocations within grains and grain rotation in the severely strained region closest to the indenter tip. In the less strained region (located further from the indenter tip) they observed the presence of cavities, which was attributed to unaccommodated GB sliding. The presence of shear bands and microcracks (formed near shear bands) was observed both in the severely strained and less strained regions. The localized deformation realized via shear banding and GB sliding as well as the formation of cracks were more pronounced in the specimen with a smaller grain size $(22 \mathrm{~nm})$. The hardness of the specimen with a smaller grain size of $22 \mathrm{~nm}$ (18.79 GPa) was also slightly lower than that of the specimen with a larger grain size of $43 \mathrm{~nm}(19.04 \mathrm{GPa})$, implying an inverse Hall-Petch relation for hardness of nanocrystalline $\mathrm{MgAl}_{2} \mathrm{O}_{4}$ spinels.

In addition to the above experimental studies and atomistic simulations, various mechanical models have been proposed to predict or explain the inverse HallPetch behavior of NCCs. For example, Jiang and Weng [37] considered NCCs as multiphase composites that are comprised of the intragrain regions, GB phase and pores. In their model, the Young modulus and yield strength of the GB phase are supposed to be much smaller than in the intragrain regions. Within such a model, using a complicated averaging procedure, they calculated the effective compressive yield strength. In a similar manner, Ehre and Chaim [12] assumed that the GB phase has smaller hardness than the internal regions of grains and used the mixture rule to calculate the hardness of the composite. As a result, Jiang and Weng [37] and Ehre and Chaim [12] obtained an inverse Hall-Petch dependence for compressive yield strength [37] or microhardness [12] below some critical grain size, for the case where the yield strength of the GB phase is chosen to be sufficiently small. (Much earlier mixturerule-based models were suggested for nanocrystalline metals (NCMs), see Refs. [19,21] for details. For the reasons of the assumed diminishing strength of the GB phase, those earlier models indicated high local porosity [38] and amorphous structure [39,40] of GBs, possible quasi-periodicity of GBs [41], the presence of triple junction disclinations [42], and the high diffusional mass transfer along GBs [43].) However, the phenomenological composite models are not physically validated, and, besides, do not explain the contradictions in the experimental data on the hardness of nanocrystalline $\mathrm{MgAl}_{2} \mathrm{O}_{4}$.

While the models describing the inverse Hall-Petch dependence in NCCs are limited to composite ones, many other models have been suggested to explain the break- down of the Hall-Petch relation in NCMs [17-31, 38-52]. Several models are based on the interaction of lattice dislocations with GBs [44-46] and assume softening of NCMs due to the existence of dislocation networks at GBs [46] or change in the excess volume of the GB phase [45]. Other models are based on various deformation mechanisms that are assumed to prevail in NCMs with ultrafine grains, which include rotational deformation carried out by the motion of triple junction disclinations [47], diffusion Coble creep over GBs [17,43,48,49], GB sliding via thermally activated local shear events [50] or glide of GB dislocations [51] and dislocation emission from triple junctions [52].

Due to a large number of models describing the breakdown of the Hall-Petch dependence in NCMs, it is tempting to extend some of these models to explain the softening of NCCs. In choosing the appropriate model, one should note that triple junction disclinations have not been documented in NCCs. Therefore, the applicability of the model considering the motion of triple junction disclinations [47] to NCCs is questionable. The models $[17,43,48,49]$ assuming the Coble creep are also hardly applicable to room temperature deformation of NCCs, since GB diffusion should be absent in ceramics at room temperature. At the same time, computer simulations $[16,34,35]$ and experimental observations [16] of plastic deformation of NCCs revealed an important role of GBs in this process. Based on the above, the concept of thermally activated GB sliding [50] have been recently used in [53] to explain both strengthening and softening of NCCs.

To do so, following [50], the authors of [53] assumed that GB sliding is initiated through the thermally activated shear events, which can be considered as the homogeneous nucleation of glide dislocation loops with the size equal to the interatomic distance. The coalescence of such small dislocation loops can result in the formation and expansion of large dislocation loops (illustrated as dislocation dipoles in the two-dimensional

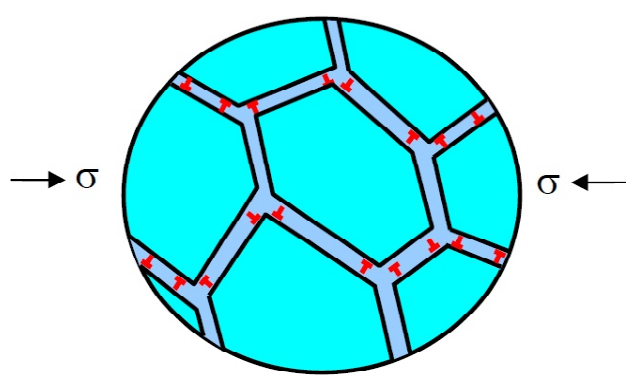

Fig. 4. A fragment of a nanocrystalline ceramic solid (under a uniform compressive stress $\sigma$ ) where plastic deformation is realized through thermally activated grain boundary sliding. Reproduced from Sheinerman et al. [53], with permission. Copyright (2020), Elsevier. 
scheme in Fig. 4) that carry GB sliding. In this situation, for a preset GB, the resolved shear stress $\tau$ can be related to the shear strain rate $\dot{\gamma}$ as [50]

$$
\dot{\gamma}=\frac{6 b v_{D}}{d} \sinh \frac{\tau b^{3}}{k_{B} T} \exp \left(-\frac{\Delta F}{R T}\right),
$$

where $b$ is the interatomic distance, $v_{D}=10^{13} \mathrm{~s}^{-1}$ is the Debye frequency, $d$ is the grain size, $k_{B}$ is the Boltzmann constant, $R$ is the universal gas constant, $T$ is the absolute temperature, and $\Delta F$ is the activation energy for thermally activated GB sliding. Since for NCCs deformed at room temperature, $\tau b^{3} /\left(k_{B} T\right)>>1$, we have: $\sinh \left[\tau b^{3} /\left(k_{B} T\right)\right] \approx(1 / 2) \exp \left[\tau b^{3} /\left(k_{B} T\right)\right]$. Using the latter relation, the equality $R=k_{B} N_{A}$, where $N_{A}=6.02 \times 10^{23}$ $\mathrm{mol}^{-1}$ is the Avogadro constant, and the relation $\sigma_{G B S}=$ $M \tau$ between the yield strength $\sigma_{G B S}$ associated with GB sliding and the shear stress $\tau$, from formula (1) one obtains [53]:

$$
\sigma_{G B S}=\frac{M}{b^{3}}\left(\frac{\Delta F}{N_{A}}+k_{B} T \ln \frac{\dot{\gamma} d}{3 b v_{D}}\right) .
$$

Within the model [53], in large enough grains, plastic deformation is realized through lattice dislocation slip, which was observed experimentally at room temperature in ceramics [54]. The critical stress $\sigma_{H P}$ for the onset of such slip is described by the Hall-Petch relation:

$$
\sigma_{H P}=\sigma_{0}+k_{H P} d^{-1 / 2},
$$

where $\sigma_{0}$ and $k_{H P}$ are constants. Following the approach of Masumura et al. [48], the authors of [53] assumed that each grain deforms by GB sliding if $\sigma_{G B S}<\sigma_{H P}$ and via intragrain dislocation motion otherwise. Then the critical (minimum) stress $\sigma_{y s}$ for the onset of plastic deformation in a given grain can be defined as $\sigma_{y s}=$ $\min \left\{\sigma_{H P}, \sigma_{G B S}\right\}$.

To take the effect of a grain size distribution into account, following [48], the authors of [53] assumed that the grain size $d$ obeys the lognormal distribution with the mean grain size $\bar{d}$, described by the probability density:

$$
f(d)=\frac{1}{d \sqrt{2 \pi s^{2}}} \exp \left[-\frac{\left(\ln (d / \bar{d})+s^{2} / 2\right)^{2}}{2 s^{2}}\right],
$$

where $s$ denotes the dispersion of $\ln d$. The macroscopic yield stress $\bar{\sigma}_{y s}$ can be calculated as a weighted average of the yield stresses of various grains [48], that is,

$$
\bar{\sigma}_{y s}=\frac{<\sigma_{y s} d^{3}>}{<d^{3}>}=\frac{\int_{0}^{\infty} f(d) \sigma_{y s} d^{3} \mathrm{~d} d}{\int_{0}^{\infty} f(d) d^{3} \mathrm{~d} d} .
$$

In formula (5), the denotation $\mathrm{d} d$ means the differential of grain size $d$.

Using formula (5), one can calculate the macroscopic yield strength $\bar{\sigma}_{y s}$ of the ceramic solid and its microhardness $H=3 \bar{\sigma}_{y s}$. To do so, for the case of $\mathrm{MgAl}_{2} \mathrm{O}_{4}$ the following parameter values have been used [53]: $s=0.2, b=0.286 \mathrm{~nm}, \sigma_{0}=4.2 \mathrm{GPa}, T=300 \mathrm{~K}$, $k_{H P}=0.48 \mathrm{MPa} \times \mathrm{m}^{1 / 2}, a=0.808 \mathrm{~nm}$, and $M=3.06$. The dependences of $H$ on $\bar{d}$ are presented in Fig. 5, for various values of the parameter $\Delta F$. As is seen in Fig. 5, the calculated values of the hardness of the nanocrystalline $\mathrm{MgAl}_{2} \mathrm{O}_{4}$ spinels are in satisfactory agreement with experimental data $[8,14,15,32]$ and demonstrate the transition from the direct to the inverse Hall-Petch dependences near a critical grain size. This critical grain size depends on the activation energy $\Delta F$ of GB sliding and corresponds to the transition in the dominant deformation mechanism from dislocation motion across grains to GB sliding.

Thus, within the model [53], there always exists a transition from the direct to the inverse Hall-Petch relation at small grain sizes. The critical grain size for this transition decreases with a decrease in the activation energy $\Delta F$ of GB sliding, which can depend on whether the GB is crystalline or amorphous, as well as on the structure of GBs and their chemical composition.

At the same time, although the model [53] explains the contradictive data for hardness of $\mathrm{MgAl}_{2} \mathrm{O}_{4}$, it fails to explain very small values of strain rate sensitivity of hardness recently documented by Ryou et al. [15]. Indeed, according to Ryou et al. [15], the strain rate sensitivity of nanocrystalline $\mathrm{MgAl}_{2} \mathrm{O}_{4}$ is at most several hundredths, while the model [53] predicts the strain rate sensitivity of several tenths typical of GB sliding in metals. Therefore, one can assume that in addition to GB sliding itself, the process of amorphization of dislocation delocalization in amorphous GBs can also be re-

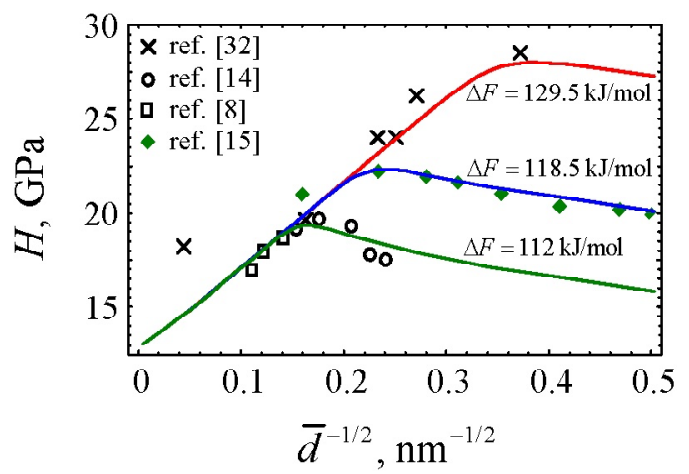

Fig. 5. Microhardness $H$ as a function of the inverse square root of the grain size, $d^{-1 / 2}$, for various values of the activation energy $\Delta F$. Reproduced from Sheinerman et al. [53], with permission. Copyright (2020), Elsevier. 
sponsible for the contradictive data on microhardness of NCCs. However, further research is required to reveal the influence of these processes on plastic deformation and hardness of nanoceramics.

Thus, from the above brief review it follows that the GB mediated processes, such as GB sliding, grain rotation and GB amorphization in NCCs can be responsible for their softening observed in experiments. Apparently, the structure and composition of GBs can dramatically influence the strength and hardness of NCCs. However, further research is required to connect the atomic-level deformation mechanisms and mechanical properties of nanoceramics.

\section{ACKNOWLEDGMENTS}

This work is supported by the Russian Science Foundation (grant 18-19-00255).

\section{REFERENCES}

[1] M.J. Mayo, D.C. Hague, and D.J. Chen, Processing nanocrystalline ceramics for applications in superplasticity, Mater. Sci. Eng. A, 1993, vol. 166, no. 1-2, pp. 145-159. https://doi.org/10.1016/09215093(93)90318-9

[2] M.J. Mayo, Processing of nanocrystalline ceramics from ultrafine particles, Intern. Mater. Rev., 1996, vol. 41, no. 3, pp. 85-115. https:// doi.org/10.1179/imr.1996.41.3.85

[3] C. Kleinlogel and L.J. Gauckler, Sintering of nanocrystalline $\mathrm{CeO}_{2}$ ceramics, Adv. Mater., 2001, vol. 13, no. 14, pp. 1081-1085. https://doi.org/ 10.1002/1521-4095(200107)13:14<1081::AIDADMA1081>3.0.CO;2-D

[4] S.J. Kalita, A. Bhardwaj, and H.A. Bhatt, Nanocrystalline calcium phosphate ceramics in biomedical engineering, Mater. Sci. Eng. C, 2007, vol. 27, no. 3, pp. 441-449. https://doi.org/10.1016/ j.msec.2006.05.018

[5] F.Y. Cheng, J.A. Shen, B. Peng, Y.D. Pan, Z.L. Tao, and J. Chen, Rapid room-temperature synthesis of nanocrystalline spinels as oxygen reduction and evolution electrocatalysts, Nature Chemistry, 2011, vol. 3, no. 1, pp. 79-84. doi: 10.1038/ nchem.931

[6] V.R. Mudinepalli, L. Feng, W.C. Lin, and B.S. Murty, Effect of grain size on dielectric and ferroelectric properties of nanostructured $\mathrm{Ba}_{0.8} \mathrm{Sr}_{0.2} \mathrm{TiO}_{3}$ ceramics, J. Adv. Ceramics, 2015, vol. 4, no. 1, pp. 46-53. https://doi.org/10.1007/s40145015-0130-8

[7] J.A. Wollmershauser, B.N. Feigelson, E.P. Gorzkowski, C.T. Ellis, R. Goswami, S.B. Qadri, J.G.
Tischler, F.J. Kub, and R.K. Everett, An extended hardness limit in bulk nanoceramics, Acta Mater., 2014, vol. 69, pp. 9-16. https://doi.org/10.1016/ j.actamat.2014.01.030

[8] M. Sokol, M. Halabi, S. Kalabukhov, and N. Frage, Nano-structured $\mathrm{MgAl}_{2} \mathrm{O}_{4}$ spinel consolidated by high pressure spark plasma sintering (HPSPS), J. Europ. Ceram. Soc., 2017, vol. 37, no. 2, pp. 755 762. https://doi.org/10.1016/ j.jeurceramsoc.2016.09.037

[9] M. Sokol, S. Kalabukhov, R. Shneck, E. Zaretsky, and N. Frage, Effect of grain size on the static and dynamic mechanical properties of magnesium aluminate spinel $\left(\mathrm{MgAl}_{2} \mathrm{O}_{4}\right)$, J. Europ. Ceram. Soc., 2017, vol. 37, no. 10, pp. 3417-3424. https:// doi.org/10.1016/j.jeurceramsoc.2017.04.025

[10] H. Wang, A. Sharma, A. Kvit, and Q. Wei, Mechanical properties of nanocrystalline and epitaxial TiN films on (100) silicon, J. Mater. Res., 2001, vol. 16, pp. 2733-2738. https://doi.org/ 10.1557/JMR.2001.0373

[11] Y. Wang, J. Zhang, and Y. Zhao, Strength weakening by nanocrystals in ceramic materials, Nano Lett., 2007, vol. 7, no. 10, pp. 2-5. https://doi.org/10.1021/n10718723

[12] D. Ehre and R. Chaim, Abnormal Hall-Petch behavior in nanocrystalline $\mathrm{MgO}$ ceramic, J. Mater. Sci., 2008, vol. 43, no. 18, pp. 6139-6143. https://doi.org/10.1007/s10853-008-2936-Z

[13] Z.B. Qi, P. Sun, F.P. Zhu, Z.C. Wang, D.L. Peng, and C.H. Wu, The inverse Hall-Petch effect in nanocrystalline ZrN coatings, Surf. Coat. Tech., 2011, vol. 205, pp. 3692-3697. https://doi.org/ 10.1016/j.surfcoat.2011.01.021

[14] M. Sokol, M. Halabi, Y. Mordekovitz, S. Kalabukhov, S. Hayun, and N. Frage, An inverse Hall-Petch relation in nanocrystalline $\mathrm{MgAl}_{2} \mathrm{O}_{4}$ spinel consolidated by high pressure spark plasma sintering (HPSPS), Scripta Mater., 2017, vol. 139, pp. 159-161. https://doi.org/ 10.1016/j.scriptamat.2017.06.049

[15] H. Ryou, J.W. Drazin, K.J. Wahl, S.B. Qadri, E.P. Gorzkowski, B.N. Feigelson, and J.A. Wollmershauser, Below the Hall-Petch limit in nanocrystalline ceramics, ACS Nano, 2018, vol. 12, no. 4, pp. 3083-3094. https://doi.org/10.1021/ acsnano. $7 \mathrm{~b} 07380$

[16] D. Guo, S. Song, R. Luo, W.A. Goddard, III, M. Chen, K.M Reddy, and Q. An, Grain boundary sliding and amorphization are responsible for the reverse Hall-Petch relation in superhard nanocrystalline boron carbide, Phys. Rev. Lett., 2018, vol. 121, 
pp. 145504(1)-145504(6). https://doi.org/10.1103/ PhysRevLett.121.145504

[17] A.H. Chokshi, A. Rosen, J. Karch, and H. Gleiter, On the validity of the Hall-Petch relationship in nanocrystalline materials, Scripta Metall., 1989, vol. 23, no. 10, pp. 1679-1684. https://doi.org/ 10.1016/0036-9748(89)90342-6

[18] V.G. Gryaznov and L.I. Trusov, Size effects in micromechanics of nanocrystals, Prog. Mater. Sci., 1993, vol. 37, no. 4, pp. 289-401. https:// doi.org/10.1016/0079-6425(93)90001-2

[19] M.Yu. Gutkin, I.A. Ovid'ko, and C.S. Pande, Theoretical models of plastic deformation processes in nanocrystalline materials, Rev. Adv. Mater. Sci., 2001, vol. 2, no. 1, pp. 80-102. http://www.ipme.ru/e-journals/RAMS/no_1201/ ovidko/ovidko.pdf

[20] S. Takeuchi, The mechanism of the inverse HallPetch relation of nanocrystals, Scripta Mater., 2001, vol. 44, pp. 1483-1487. https://doi.org/ 10.1016/S1359-6462(01)00713-8

[21] M.Yu. Gutkin and I.A. Ovid'ko, Plastic deformation in nanocrystalline materials, Springer, Berlin/Heidelberg/New York, 2004.

[22] M.A. Meyers, A. Mishra, and D.J. Benson, Mechanical properties of nanocrystalline materials, Prog. Mater. Sci., 2006, vol. 51, no. 4, pp. 427-556. https://doi.org/10.1016/ j.pmatsci.2005.08.003

[23] G.A. Malygin, Plasticity and strength of microand nanocrystalline materials, Phys. Solid State, 2007, vol. 49, no. 6, pp. 1013-1033. https://doi.org/ $10.1134 / \mathrm{S} 1063783407060017$

[24] R.A. Andrievski and A.M. Glezer, Strength of nanostructures, Physics - Uspekhi, 2009, vol. 52, no. 4, pp. 315-334. https://doi.org/10.3367/ UFNe.0179.200904a.0337

[25] C.S. Pande and K.P. Cooper, Nanomechanics of Hall-Petch relationship in nanocrystalline materials, Prog. Mater. Sci., 2009, vol. 54, no. 6, pp. 689-706. https://doi.org/10.1016/ j.pmatsci.2009.03.008

[26] D.J. Dunstan and A.J. Bushby, Grain size dependence of the strength of metals: The HallPetch effect does not scale as the inverse square root of grain size, Int. J. Plasticity, 2014, vol. 53, pp. 56-65. https://doi.org/10.1016/ j.ijplas.2013.07.004

[27] E.N. Hahn and M.A. Meyers, Grain-size dependent mechanical behavior of nanocrystalline metals, Mater. Sci. Eng. A, 2015, vol. 646, pp. 101-134. https://doi.org/10.1016/ j.msea.2015.07.075
[28] E.V. Kozlov, A.M. Glezer, N.A. Koneva, N.A. Popova, and N.A. Kurzina, Fundamentals of plastic deformation of nanostructured materials, Fizmatlit, Moscow, 2016, In Russian.

[29] Z.C. Cordero, B.E. Knight, and C.A. Schuh, Six decades of the Hall-Petch effect-a survey of grain-size strengthening studies on pure metals, Int. Mater. Rev., 2016, vol. 61, pp. 495-512. https:// doi.org/10.1080/09506608.2016.1191808

[30] R.W. Armstrong, Dislocation pile-ups, strength properties and fracturing, Rev. Adv. Mater. Sci., 2017, vol. 48, no. 1, pp. 1-12. http://www.ipme.ru/ e-journals/RAMS/no_14817/ 01_14817_armstrong.pdf

[31] L. Tian, A short review on mechanical behavior of nanocrystalline materials, Int. J. Metall. Met. Phys., 2017, vol. 2, pp.008(1)-008(13). https:// doi.org/10.35840/2631-5076/9208

[32] D.N.F. Muche, J.W. Drazin, J. Mardinly, S. Dey, and R.H.R. Castro, Colossal grain boundary strengthening in ultrafine nanocrystalline oxides, Mater. Lett., 2017, vol. 186, pp. 298-300. https://doi.org/10.1016/j.matlet.2016.10.035

[33] C. Yang, A. Thron, and R.H.R. Castro, Grain boundary strengthening in nanocrystalline zinc aluminate, J. Amer. Cer. Soc., 2019, vol. 102, pp. 6904-6912. https://doi.org/10.1111/jace.16512

[34] I. Szlufarska, A. Nakano, and P. Vashishta, A crossover in the mechanical response of nanocrystalline ceramics, Science, 2005, vol. 309, no. 5736, pp. 911-914. https://doi.org/10.1126/ science. 1114411

[35] Q. Feng, X. Song, X. Liu, S. Liang, H. Wang, and Z. Nie, Compression deformation of WC: atomistic description of hard ceramic material, Nanotechnology, 2017, vol. 28, no. 47, pp. 475709(1)-475709(8). https://doi.org/10.1088/13616528/aa9270

[36] B. Ratzker, A. Wagner, M. Sokol, L. Meshi, S. Kalabukhova, and N. Frage, Deformation in nanocrystalline ceramics: A microstructural study of $\mathrm{MgAl}_{2} \mathrm{O}_{4}$, Acta Mater., 2020, vol. 183, pp. 137-144. https://doi.org/10.1016/ j.actamat.2019.11.015

[37] B. Jiang and G.J. Weng, A theory of compressive yield strength of nano-grained ceramics, Int. J. Plasticity, 2004, vol. 20, no. 11, pp. 2007-2026. https://doi.org/10.1016/j.ijplas.2003.10.010

[38] V.G. Gryaznov, M.Yu. Gutkin, A.E. Romanov, and L.I. Trusov, On the yield stress of nanocrystals, J. Mater. Sci., 1994, vol. 28, no. 16, pp. 4359-4365. https://doi.org/10.1007/BF01154943 
[39] J.E. Carsley, J. Ning, W.W. Milligan, S.A. Hackney, and E.C. Aifantis, A simple, mixturesbased model for the grain size dependence of strength in nanophase metals, Nanostruct. Maters., 1995, vol. 5, no. 4, pp. 441-448. https:// doi.org/10.1016/0965-9773(95)00257-F

[40] H.S. Kim, A composite model for mechanical properties of nanocrystalline materials, Scripta Mater., 1998, vol. 39, no. 8, pp. 1057-1061. https:// doi.org/10.1016/j.pmatsci.2005.08.003

[41] I.A. Ovid'ko, Quasi-nanocrystalline materials, Nanostruct. Maters., 1997, vol. 8, no. 2, pp. 149-153. https://doi.org/10.1016/S09659773(97)00006-8

[42] D.A. Konstantinidis and E.C. Aifantis, On the "anomalous" hardness of nanocrystalline materials, Nanostruct. Maters., 1998, vol. 10, no. 7, pp. 1111-1118. https://doi.org/10.1016/S09659773(98)00145-7

[43] H.S. Kim, Y. Estrin, and M.B. Bush, Plastic deformation behavior of fine grained materials, Acta Mater., 2000, vol. 48, no. 2, pp. 493-504. https://doi.org/10.1016/S1359-6454(99)00353-5

[44] G.A. Malygin, Breakdown of the Hall-Petch law in micro- and nanocrystalline materials, Phys. Solid State, 1995, vol. 37, no. 8, pp. 1248-1253.

[45] K. Lu and M.L. Sui, An explanation to the abnormal Hall-Petch relation in nanocrystalline materials, Scripta Metall. Mater., 1993, vol. 28, no. 12, pp. 1465-1470. https:// doi.org/10.1016/0956-716X(93)90576-E

[46] R.O. Scattergood and C.C. Koch, A modified model for Hall-Petch behavior in nanocrystalline materials, Scripta Metall. Mater., 1992, vol. 27, no. 9, pp. 1195-1200. https://doi.org/ 10.1016/0956-716X(92)90598-9

[47] S.G. Zaichenko and A.M. Glezer, Disclination mechanism for plastic deformation of nanocrystalline materials, Phys. Solid State,
1997, vol. 39, no. 11, pp. 1810-1814. https:// doi.org/10.1134/1.1130179

[48] R.A. Masumura, P.M. Hazzledine, and C.S. Pande, Yield stress of fine grained materials, Acta Mater., 1998, vol. 46, no. 13, pp. 4527-4534. https:/ /doi.org/10.1016/S1359-6454(98)00150-5

[49] A.A. Fedorov, M.Y. Gutkin, and I.A. Ovid'ko, Triple junction diffusion and plastic flow in finegrained materials, Scripta Mater., 2002, vol. 47, no. 1, pp. 51-55. https://doi.org/10.1016/S13596462(02)00096-9

[50] H. Conrad and J. Narayan, On the grain size softening in nanocrystalline materials, Scripta Mater., 2000, vol. 42, no. 11, pp. 1025-1030. https:/ /doi.org/10.1016/S1359-6462(00)00320-1

[51] M.Yu. Gutkin, I.A. Ovid'ko, and C.S. Pande, Yield stress of nanocrystalline materials: Role of grain boundary dislocations, triple junctions and Coble creep, Philos. Mag., 2004, vol. 84, no. 9, pp. 847-863. https://doi.org/10.1080/ 14786430310001616063

[52] S.S. Quek, Z.H. Chooi, Z. Wu, Y.W. Zhang, and D.J. Srolovitz, The inverse hall-petch relation in nanocrystalline metals: A discrete dislocation dynamics analysis, J. Mech. Phys. Solids, 2016, vol. 88, pp. 252-266. https://doi.org/10.1016/ j.jmps.2015.12.012

[53] A.G. Sheinerman, R.H.R. Castro, and M.Yu. Gutkin, A model for direct and inverse HallPetch relation for nanocrystalline ceramics, Mater. Lett., 2020, vol. 260, pp. 126886(1)126886(3). https://doi.org/10.1016/ j.matlet.2019.126886

[54] S. Kondo, T. Mitsuma, N. Shibata, and Y. Ikuhara, Direct observation of individual dislocation interaction processes with grain boundaries, Science Advances, 2016, vol. 2, pp. e1501926(1)e1501926(7). https://doi.org/10.1126/ sciadv.1501926 